Received: 22.10 .2018

Revised: 15.11 .2018

Accepted: 30.11 .2018

DOI: $10.17804 / 2410-9908.2018 .6 .090-099$

\title{
APPLICATION OF THE INSTRUMENTED NANOINDENTATION METHOD TO EVALUATING THE BEHAVIOR OF THE MECHANICAL PROPERTIES OF A Fe-Ga ALLOY WITH INCREASING GALLIUM CONTENT
}

\author{
V. A. Milyutin ${ }^{\mathrm{a})^{*}}$ and I. V. Gervasieva ${ }^{\text {b) }}$ \\ M.N. Miheev Institute of Metal Physics, Ural Branch of the Russian Academy of Sciences, \\ 18 S. Kovalevskoy St., Ekaterinburg, 620108, Russian Federation \\ a) (iD https://orcid.org/0000-0002-5808-3959 milutin@imp.uran.ru; \\ b) iD https://orcid.org/0000-0001-8928-1707 $\otimes$ gervasy@imp.uran.ru \\ *Corresponding author. E-mail: v.a.milutin@gmail.com \\ Address for correspondence: ul. S. Kovalevskoy, 18, Ekaterinburg, 620108, Russia
}

Tel.: +73433783820

Five Fe-Ga samples with gallium content ranging from 13.2 to 19.7 at. $\%$ are studied in this paper. X-ray phase analysis is carried out for the certification of the phase composition of the initial samples; in order to find differences in the chemical composition inside the grains and in the nearborder areas, elementary analysis of individual structure sections is carried out. It is demonstrated that in this alloy there is a distinct brittle fracture. To study the mechanical properties and to reveal their behavior with increasing Ga content, it is proposed to use a nanoindentation system. An increase in microhardness and a decrease in the plastic work of indentation with increasing Ga content are shown.

Keywords: Fe-Ga alloy, instrumented microindentation, fracture.

\section{Acknowledgment}

The work was performed within the state assignment from FASO Russia (Magnet, No. AAAA-A18-118020290129-5) and partially supported by the RFBR (project No. 18-03-00623) and UB RAS Complex Program No. 18-10-2-5.

The experimental results were obtained with the use of the equipment of the Testing Center of Nanotechnology and Advanced Materials collective use center.

\section{References}

1. The experimental results were obtained with the use of the equipment of the Testing Center of Nanotechnology and Advanced Materials collective use centerClark A.E., Restorff J.B., Wun-Fogle M., Lograsso T.A., Schlagel D.L. Magnetostrictive properties of body-centered cubic Fe-Ga and Fe-Ga-Al alloys. IEEE Transactions on Magnetics, 2000, vol. 36, pp. 3238-3240. DOI: $10.1109 / 20.908752$.

2. $\quad$ Xing Q., Du Y., McQueeney R.J., Lograsso T.A. Structural investigations of Fe-Ga alloys: Phase relations and magnetostrictive behavior. Acta Materialia, 2008, vol. 56, pp. 4536-4546. DOI: 10.1016/j.actamat.2008.05.011

3. Na S., Flatau A.B. Single grain growth and large magnetostriction in secondarily recrystallized Fe-Ga thin sheet with sharp Goss (011) [100] orientation. Scripta Materialia, 2012, vol. 66, pp. 307-310. DOI: 10.1016/j.scriptamat.2011.11.020. 
4. Clark A.E., Wun-Fogle M., Restorff J.B., Lograsso T.A. Magnetostrictive properties of galfenol alloys under compressive stress. Materials Transaction, 2002, vol. 43, pp. 881-886. DOI: $10.2320 /$ matertrans.43.881.

5. Kellogg R.A., Flatau A.B., Clark A.E., Wun-Fogle M., Lograsso T.A. Temperature and stress dependencies of the magnetic and magnetostrictive properties of Fe $0.81 \mathrm{Ga} 0.19$. J. Appl. Phys., 2002, vol. 91, pp. 7821-7823. DOI: 10.1063/1.1452216.

6. Jayaraman T.V., Srisukhumbowornchai N., Guruswamy S., Free M.L. Corrosion studies of single crystals of iron-gallium alloys in aqueous environments. Corros. Sci., 2007, vol. 49, pp. 4015-4027. DOI: 10.1016/j.corsci.2007.05.010.

7. Golovin I.S., Riviure A. Mechanisms of anelasticity in Fe-13Ga alloy. Intermetallics, 2011, vol. 19, pp. 453-459. DOI: 10.1016/j.intermet.2010.10.017.

8. Golovin I.S. Anelasticity of Fe-Ga based alloys. Materials \& Design, 2015, vol. 88, pp. 577-587. DOI: 10.1016/j.matdes.2015.08.160.

9. Xing Q., Lograsso T.A. Effect of cooling rate on magnetoelasticity and short-range order in Fe-Ga alloys. Scripta Materialia, 2011, vol. 65, pp 359-362. DOI: 10.1016/j.scriptamat.2011.05.010.

10. Li J.H., Gao X.X., Zhu J., Bao X.Q., Xia T., Zhang M.C. Ductility, texture and large magnetostriction of Fe-Ga-based sheets. Scripta Materialia, 2010, vol. 63, pp. 246-249. DOI: 10.1016/j.scriptamat.2010.03.068.

11. Na S., Flatau A.B. Deformation behavior and magnetostriction of polycrystalline $\mathrm{Fe}-\mathrm{Ga}-\mathrm{X}$ (X = B, C, Mn, Mo, Nb, NbC) alloys. Journal of Applied Physics, 2008, vol. 103, pp. $07 \mathrm{D} 304$. DOI: $10.1063 / 1.2838772$.

12. Li J., Gao X., Zhu J., He C., Qiao J., Zhang W. M. Texture evolution and magnetostriction in rolled (Fe81Ga19) $99 \mathrm{Nb1}$ alloy. J. Alloys. Compd., 2009, vol. 476, pp. 529-533. DOI: 10.1016/j.jallcom.2008.09.087.

13. Li J., Gao X., Zhu J., Li Jie, Zhang M. Ductility enhancement and magnetostriction of polycrystalline Fe-Ga based alloys. J. Alloys. Compd., 2009, vol. 484, pp. 203-206. DOI: $10.1016 /$ j.jallcom.2009.03.008

14. Sun A.L., Liu J.H., Jiang C.B. Microstructural characteristics and in situ reinforcement in NbC-doped Fe81Ga19 magnetostrictive alloys. Materials \& Design, 2015, vol. 88, pp. 1342-1346. DOI: 10.1016/j.matdes.2015.08.150.

15. Takahashi T., Okazaki T., Furuya Y. Improvement in the mechanical strength of magnetostrictive (Fe-Ga-Al)-X-C (X $=\mathrm{Zr}, \mathrm{Nb}$ and $\mathrm{Mo}$ ) alloys by carbide precipitation. Scr. Mater., 2009, vol. 61, pp. 5-7. DOI: 10.1016/j.scriptamat.2008.12.032.

16. Wu Y., Fang L., Meng C., Chen Y., Wang J., Liu J., Zhang T., Jiang C. Improved magneostriction and mechanical properties in dual-phase FeGa single crystal. Materials Research Letters, 2018, vol. 6, pp. 327-332. DOI: 10.1080/21663831.2018.1451403.

17. Influence of $\mathrm{Tb}$ on structure and properties of $\mathrm{Fe}-19 \% \mathrm{Ga}$ and $\mathrm{Fe}-27 \% \mathrm{Ga}$ alloys / Golovin I.S., Balagurov E.M., Palacheva V.V., Emdadi A., Bobrikov I.A., Churyumov A.Y., Cheverikin V.V., Pozdniakov A.V., Mikhaylovskaya A.V., Golovin S.A. J. Alloys. Compd., 2016, vol. 707, pp. 51-56. DOI: 10.1016/j.jallcom.2016.09.151.

18. Zhang M.C., Jiang H.L., Gao X.X., Zhu J., Zhou S.Z. Magnetostriction and microstructure of the melt-spun Fe 83 Ga 17 alloy. Journal of Applied Physics, 2006, vol. 99, pp. 023903. DOI: $10.1063 / 1.2164528$.

19. Liu G.D., Liu L.B., Liu Z.H., Zhang M., Chen J.L., Li J.Q., Wu G.H. Giant magnetostriction on Fe85Ga15 stacked ribbon samples. Appl. Phys. Lett., 2004, vol. 84, pp. 2124-2126. DOI: $10.1063 / 1.1688452$.

20. Atulasimha J., Flatau A.B. A review of magnetostrictive iron-gallium alloys. Smart Mater. Struct., 2011, vol. 20, pp. 043001-043001. DOI: 10.1088/0964-1726/20/4/043001. 
21. Summers E.M., Lograsso T.A., Snodgrass J.D., Slaughter J.C. Magnetic and Mechanical Properties of Polycrystalline Galfenol. In: Smart Structures and Materials 2004: Active Materials: Behavior and Mechanics: Proceedings, 2004, vol. 448. DOI: 10.1117/12.539781.

22. Ikeda O., Kainuma R., Ohnuma I., Fukamichi K., Ishida K. Phase equilibria and stability of ordered b.c.c. phases in the Fe-rich portion of the $\mathrm{Fe}-\mathrm{Ga}$ system. J. Alloys. Compd., 2002, vol. 347, pp. 198-205. DOI: 10.1016/S0925-8388(02)00791-0.

23. Pineau A., Benzerga A.A., Pardoen T. Failure of metals I: Brittle and ductile fracture. Acta Materialia, 2016, vol. 107, pp. 424-483. DOI: 10.1016/j.actamat.2015.12.034.

24. Khachaturyan A.G., Viehland D. Structurally heterogeneous model of extrinsic magnetostriction for Fe-Ga and similar magnetic alloys: part I. decomposition and confined displacive transformation. Metall. Mater. Trans. A, 2007, vol. 38A, pp. 2308-2316. DOI: $10.1007 / \mathrm{s} 11661-007-9253-\mathrm{z}$.

25. Bhattacharyya S., Jinschek J.R., Khachaturyan A., Cao H., Li J.F., Viehland D. Nanodispersed DO3-phase nanostructures observed in magnetostrictive $\mathrm{Fe}-19 \% \mathrm{Ga}$ Galfenol alloys. Phys. Rev. B, 2008, vol. 77, pp. 104107. DOI: 10.1103/PhysRevB.77.104107.

26. Cao H., Gehring P.M., Devreugd C.P., Rodriguez-Rivera J.A., Li J., Viehland D. Role of Nanoscale Precipitates on the Enhanced Magnetostriction of Heat-Treated Galfenol $\left(\mathrm{Fe}_{1-\mathrm{x}} \mathrm{Ga}_{\mathrm{x}}\right)$ Alloys. Phys. Rev. Lett., 2009, vol. 102, pp. 127201. DOI: 10.1103/PhysRevLett.102.127201. 
Подана в журнал: 22.10 .2018

УДК 539.533

DOI: $10.17804 / 2410-9908.2018 .6 .090-099$

\title{
ИСПОЛЬЗОВАНИЕ МЕТОДА ИНСТРУМЕНТАЛЬНОГО НАНОИНДЕНТИРОВАНИЯ ДЛЯ ОЦЕНКИ ИЗМЕНЕНИЯ МЕХАНИЧЕСКИХ СВОЙСТВ СПЛАВА FE-GA ПРИ УВЕЛИЧЕНИИ СОДЕРЖАНИЯ ГАЛЛИЯ
}

\author{
В. А. Милютин ${ }^{\text {a)* }}$, И. В. Гервасьева ${ }^{\text {б) }}$ \\ Уральское отделение Российской Академии Наук, Институт физики металлов, \\ ул. С. Ковалевской, д. 18, Екатеринбург, Российская Федерация \\ a) (iD https://orcid.org/0000-0002-5808-3959 milutin@imp.uran.ru; \\ б) (iD https://orcid.org/0000-0001-8928-1707 gervasy@imp.uran.ru \\ *Ответственный автор. Электронная почта: v.a.milutin@gmail.com \\ Адрес для переписки: ул. С. Ковалевской, д. 18, 620108, Екатеринбург, Российская Федерация \\ Тел.: +7 (343) 378-38-20
}

В работе исследованы 5 образцов сплава Fe-Ga с содержанием галлия от 13,2 до 19,7 ат. \%. Для аттестации фазового состава образцов проведен рентгенофазовый анализ, а с целью установления различий по химическому составу в теле зерна и в приграничных областях проведен элементарный анализ отдельных участков структуры. Показано, что в данном сплаве имеет место ярко выраженный хрупкий излом. Для исследования механических свойств и установления характера их изменения при увеличении содержания Ga предложено использовать систему наноиндентирования. Показано увеличение микротвердости и уменьшение пластической работы индентирования по мере роста содержания галлия.

Ключевые слова: сплав Fe-Ga, инструментальное микроиндентирование, излом.

\section{1. Введение}

Аномально высокая магнитострикция насыщения обнаружена в сплаве железо-галлий в 2000 г. [1], с тех пор этот материал привлекает к себе повышенное внимание. За годы исследований было показано, что помимо высокой магнитострикции, которая в монокристалле может достигать порядка 400 ppm [2], этот материал обладает рядом уникальных свойств. В частности, магнитное насыщение происходит в поле напряженностью порядка 15000 A/M, что существенно ниже аналогичных значений в распространенном магнитострикционном материале Tb-Dy-Fe ( 80 000 A/м) [3]. Сплав железо-галлий имеет высокую точку кюри $\left(\sim 700{ }^{\circ} \mathrm{C}\right)[4]$ и, что тоже немаловажно при эксплуатации, слабую зависимость магнитных свойств от температуры [5]. Кроме того, данный материал обладает хорошей коррозионной стойкостью [6] и демпфирующими свойствами за счет магнитомеханической природы затухания внутреннего трения [7,8]. Все эти свойства, наряду с низкой стоимостью, делают сплав $\mathrm{Fe}-\mathrm{Ga}$ крайне привлекательным для использования в промышленности при производстве датчиков, приводов, источников и приемников ультразвука и других изделий.

В сплаве железо-галлий при увеличении содержания галлия примерно до 21 ат. \% происходит монотонное увеличение константы магнитострикции $\lambda_{100}$ [9] (рис. 1). Существенной проблемой сплава $\mathrm{Fe}-\mathrm{Ga}$ является высокая хрупкость, что затрудняет изготовление изделий и образцов. В литературе приводятся примеры того, что механическая обработка при изготовлении образцов проблематична из-за разрушения образцов, особенно при комнатной температуре $[10,11]$. В поисках решения этой проблемы, двойной сплав железогаллий предлагают легировать третьим элементом, в частности $\mathrm{B}, \mathrm{C}, \mathrm{Al}, \mathrm{V}, \mathrm{Cr}, \mathrm{Mo}, \mathrm{Nb}$ [11-13], либо добавляют карбиды $\mathrm{Zr}, \mathrm{Nb}$, Ta $[14,15]$, однако одновременно с улучшением 
механических свойств, как правило, происходит ухудшение магнитных. Относительных успехов в этом направлении удалось добиться только в последние годы с помощью легирования двойного сплава малыми добавками Тb и В $[16,17]$. Другим направлением исследований является поиск нестандартных методик изготовления образцов. В ряде работ для получения ленты предлагается использовать метод разливки на быстро вращающийся медный барабан $[18,19]$. В работе [3] показана возможность получить листы из сплава Fe-19 ат. \% Ga по технологии производства анизотропной электротехнической стали с использованием в качестве ингибиторной фазы частиц $\mathrm{NbC}$.

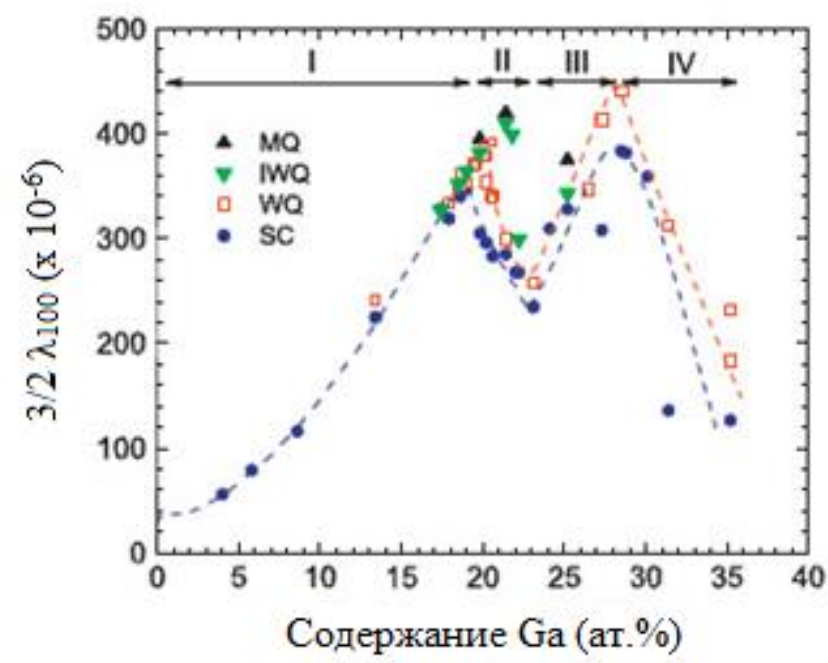

Рис. 1 . Изменение тетрагональной магнитострикции $\lambda_{100}$ с увеличением содержания $\mathrm{Ga}$ при разных режимах охлаждения: MQ - закалка в жидкий металл (GaInSn); IWQ - закалка в ледяную воду; WQ - закалка в воду; SC - медленное охлаждение [9]

Наиболее перспективным считается способ изготовления образцов $\mathrm{Fe}-\mathrm{Ga}$ с помощью зонной плавки [20], поскольку он позволяет получить образцы с острой кубической текстурой $<100>\{001\}$, что благоприятно сказывается на магнитных свойствах. Тем не менее указанные методы также имеют определенные ограничения и недостатки, прежде всего это сложная с технической точки зрения реализация и дороговизна, а также повышенные требования к чистоте сплава и защитной атмосфере. Более того, из сплава $\mathrm{Fe}-\mathrm{Ga}$ часто необходимо изготовить тонкие листы, поскольку высокая электрическая проводимость приводит к большим потерям на вихревые токи при частом перемагничивании. Изготовление тонких листов так или иначе включает процедуру прокатки на большие степени деформации, по этой причине необходимы дальнейшие поиски путей решения проблемы разрушения образцов при деформации. В то же время из-за хрупкости сплава проведение стандартных испытаний для определения механических свойств затруднительно, поскольку образцы могут ломаться даже при подготовительных операциях, например, при обтачивании. По этой причине в литературе имеются крайне ограниченные сведения по механическим свойствам $\mathrm{Fe}-\mathrm{Ga}$. Так в некоторых работах отмечается, что предел прочности для поликристалла $\mathrm{Fe}-17$ ат. \% $\mathrm{Ga}$ составляет порядка 350 МПа, а удлинение до разрыва около 1 \%. В монокристаллическом состоянии предел прочности порядка 500 МПа, удлинение до разрыва - $2 \%$ [10, 21].

В последние годы в экспериментальную практику вошел «безобразцовый» метод инструментального индентирования, который позволяет получить характеристики прочности и пластичности материалов. Метод основан на записи диаграммы вдавливания индентора в поверхность контролируемого материала в координатах «нагрузка-глубина» на упругопластической стадии нагружения и разгрузки. В настоящей работе предпринята попытка проведения сравнительного анализа упругих и пластических свойств образцов сплавов с разным со- 
держанием галлия методом инструментального микроиндентирования с целью установления влияния его концентрации на свойства и, в конечном итоге, на обрабатываемость сплава. Это позволит в дальнейшем выбрать наиболее оптимальное содержание галлия с точки зрения соотношения удовлетворительной обрабатываемости и магнитострикции.

\section{2. Материал и методика}

Образцы Fe-Ga были выплавлены в дуговой печи с использованием защитной атмосферы. Для уменьшения неравномерности состава каждый слиток был подвергнут 5-кратной переплавке и затем отлит в медную изложницу. После этого был проведен гомогенизирующий отжиг в вакууме при температуре $950{ }^{\circ} \mathrm{C}$ в течении 30 ч. В работе были исследованы 5 образцов с содержанием галлия 13,2 ат. \%; 16,8 ат. \%; 18,6 ат. \%; 19,1 ат. \%; 19,7 ат. \%. Согласно диаграмме $\mathrm{Fe}-\mathrm{Ga}$ основными фазами в сплаве с таким содержанием Ga должны быть $\mathrm{A} 2, \mathrm{DO}_{3}, \mathrm{~L}_{12}$, и $\mathrm{DO}_{22}$. Однако вследствие низкой скорости взаимодиффузии $\mathrm{Fe}$ и $\mathrm{Ga}$ формирование упорядоченных фаз происходит крайне медленно, поэтому принято пользоваться метастабильной фазовой диаграммой, согласно которой при медленном охлаждении имеет место фазовый состав $\mathrm{A} 2+\mathrm{DO}_{3}[22]$. В то же время выделение упорядоченной фазы $\mathrm{DO}_{3}$ можно подавить относительно быстрым охлаждением. Эта фаза негативным образом сказывается на свойствах, началу ее выделения соответствует область снижения магнитострикции II на рис. 1, поэтому образцы Fe-Ga, как правило, подвергают разным видам закалки или охлаждению на воздухе, чтобы исключить возможность формирования упорядоченной фазы $\mathrm{DO}_{3}$. В данной работе образцы охлаждались на воздухе, что также препятствует формированию $\mathrm{DO}_{3}$ фазы. Для того чтобы аттестовать фазовый состав, использовали рентгеновский дифрактометр Panalytical Empyrean. Дифрактограмма, полученная с одного из образцов (Fe-18,6 \% Ga), представлена на рис. 2, на котором пиков, характерных для упорядоченной сверхструктуры, не наблюдается. Аналогичные результаты получены для всех исследуемых образцов. Структура была изучена с использованием сканирующего электронного микроскопа FEI Quanta-200. Химический анализ проводили в камере микроскопа с использованием энергодисперсионного анализатора. Для сравнительного анализа упругих и пластических свойств в работе использовали комплексную систему измерений на микро- и наноуровне Nanotest 600.

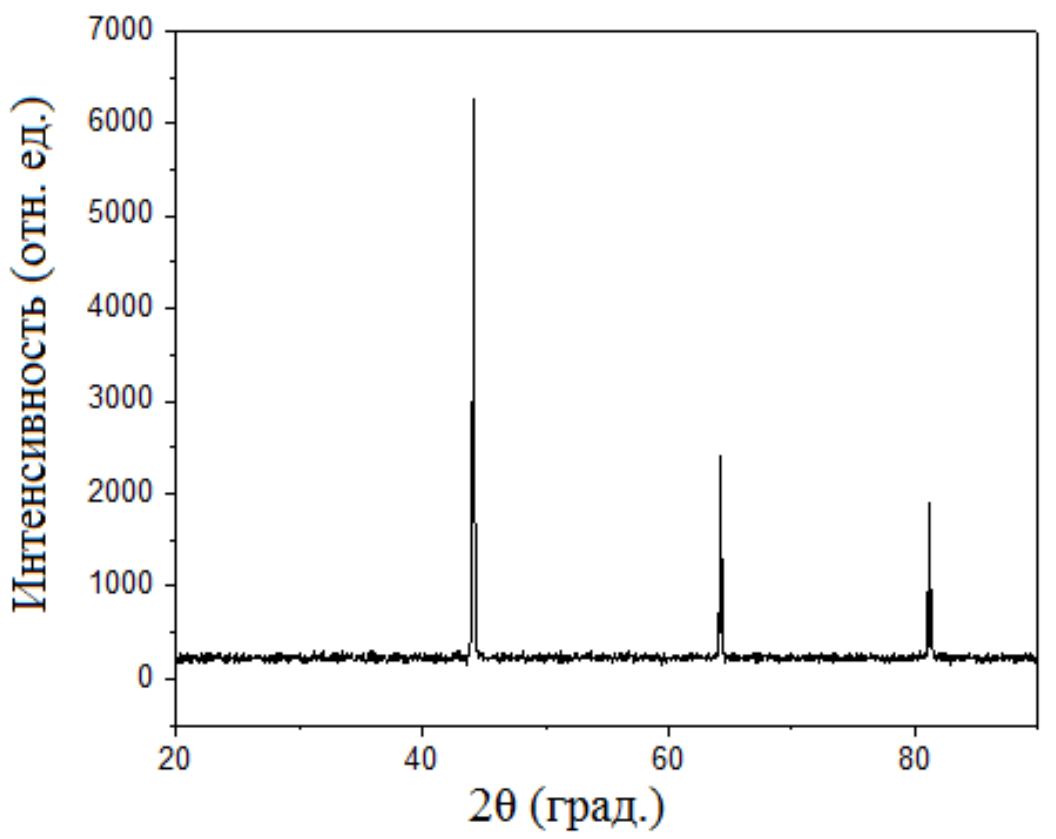

Рис. 2. Дифрактограмма, полученная с образца Fe-18,6 ат. \% Ga 


\section{3. Результаты и обсуждение}

Исходная структура всех образцов является типичной для литого двухкомпонентного сплава на основе железа и состоит из крупных зерен, равноосных в центре сечения слитка и вытянутых от центра к краям в средней части сечения. Какой-либо преимущественной кристаллографической ориентировки не выявлено. Как было отмечено во введении, образцы сплава Fe-Ga склонны разрушаться даже при незначительном механическом воздействии во время стандартных технологических операций, таких как токарная обработка. На рис. 3 представлены фотографии такого излома образца с 19,1 ат. \% Ga. Можно видеть, что имеет место типичный межзеренный хрупкий излом [23]. Механизмом разрушения является межзеренное расщепление (рис. 3 a). Такой излом характерен для материалов с минимальной пластичностью. При большем увеличении хорошо видны террасы хрупкого скола (рис. 3 б).

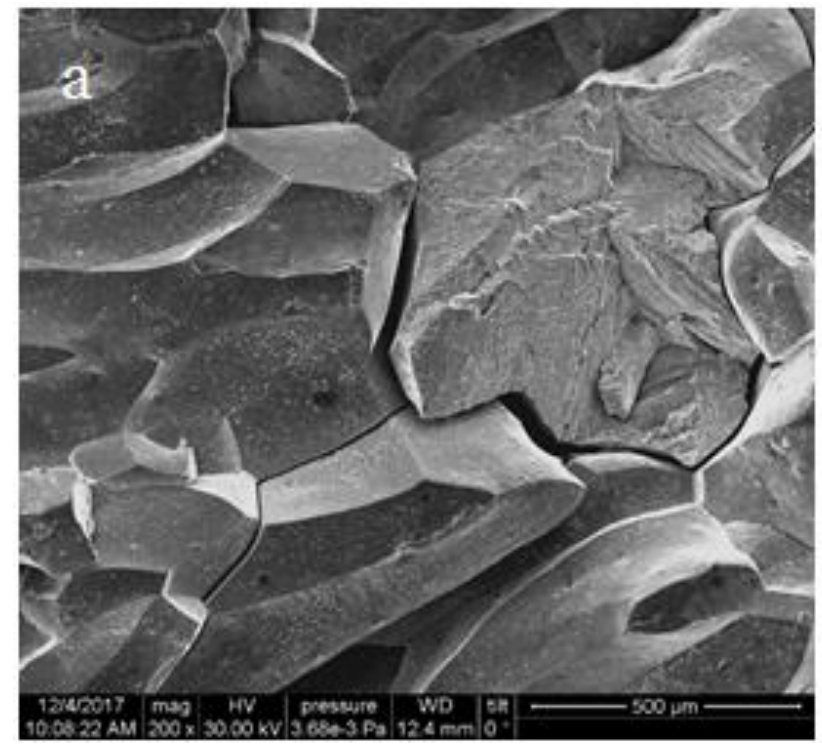

$a$

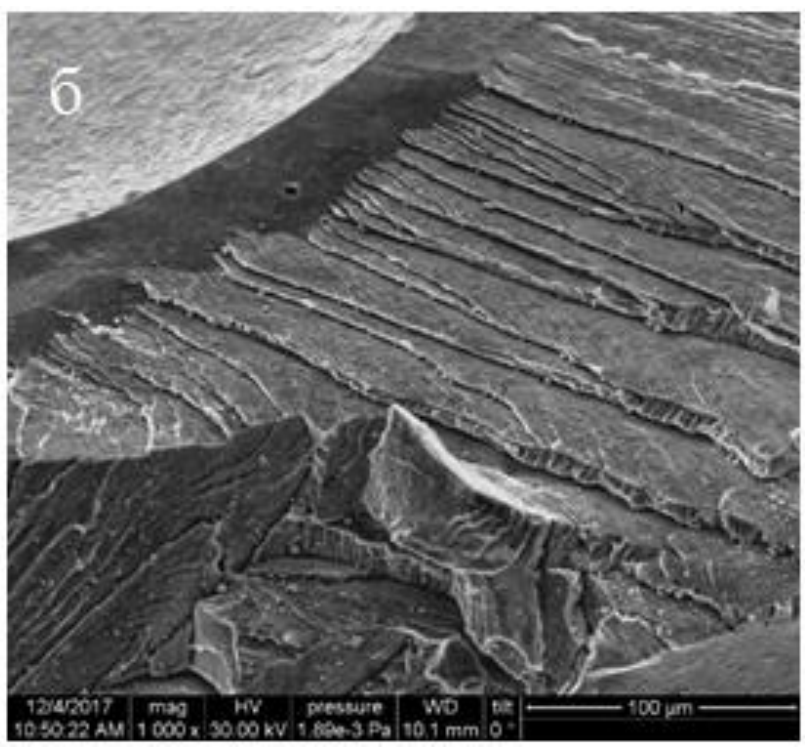

6

Рис. 3. Фотографии излома, полученные с помощью сканирующего электронного микроскопа при увеличении $\times 200(a)$ и $\times 1000$ (б)

Очевидно, что добавление $\mathrm{Ga}$ в железо оказывает охрупчивающее влияние. Как правило, такое явление связывают с сегрегацией легирующего элемента на границах зерен. Однако используемыми в данной работе методами показать существенное увеличение содержания $\mathrm{Ga}$ на границе напрямую не удалось. Тем не менее в некоторых случаях наблюдается незначительная тенденция, которая, впрочем, может лежать в пределах погрешности измерений. На рис. 4. приведен химический состав различных участков в теле зерна и в приграничных областях. При среднем содержании Ga 19 ат. \%, эта величина на границе может достигать 20,4\% (рис. 4 a).

Исследование свойств сплавов методом микроиндентирования проводилось при комнатной температуре и нагрузке 256 мН. На рис. 5 представлены кривые, соответствующие изменению глубины проникновения индентора (a), пластической работы (б) и твердости (в) с увеличением содержания Ga. Каждая точка получена путем усреднения серии индентирований. Величина «пластическая работа» соответствует площади под кривой «нагрузкаглубина» вдавливания и приводится здесь с целью сравнительной оценки пластических свойств образцов. 


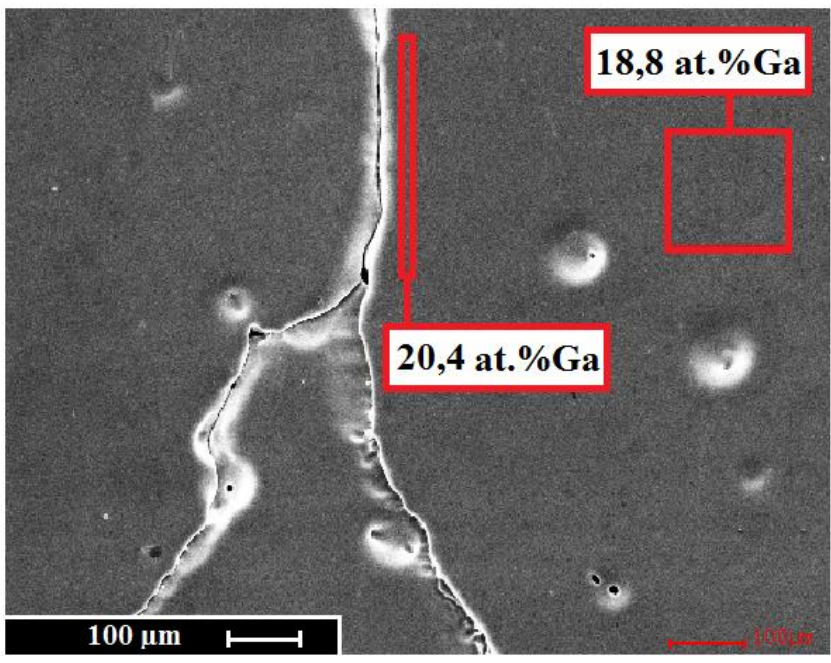

$a$

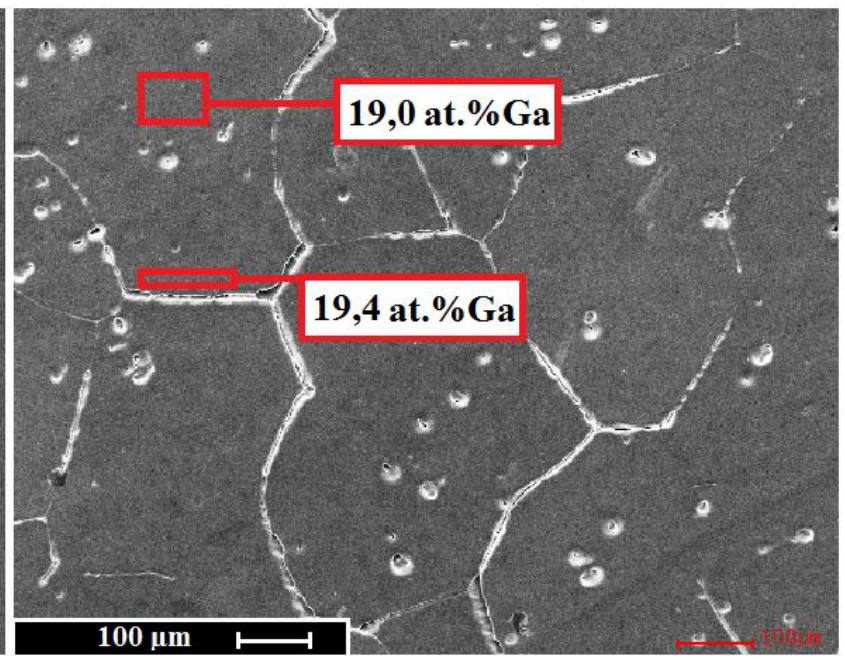

6

Рис. 4. Выборочный химический анализ отдельных участков структуры

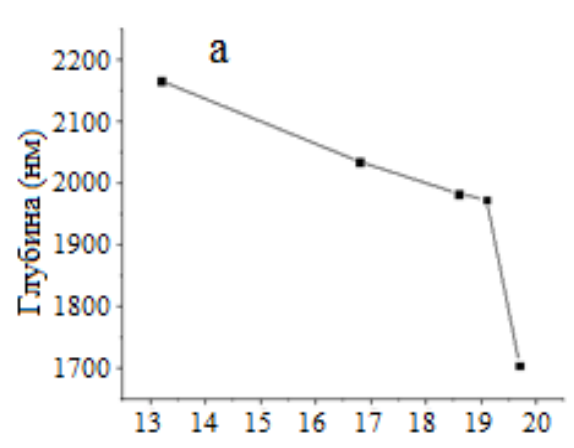

$a$

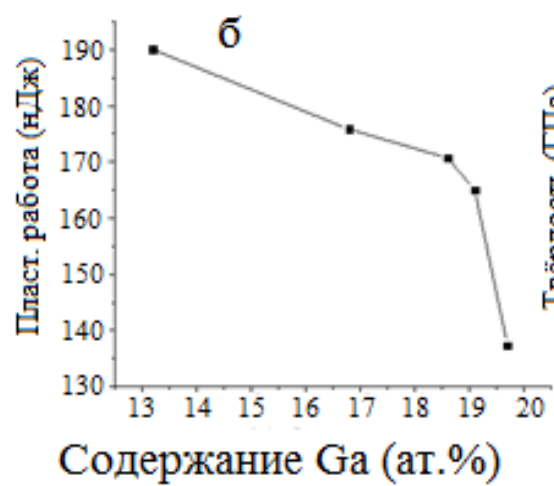

$\sigma$

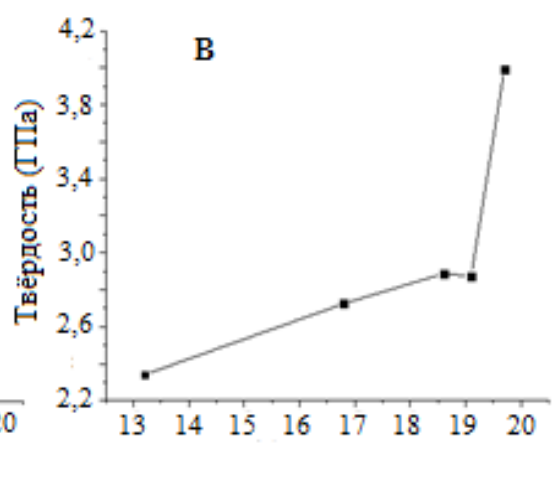

B

Рис. 5. Графики изменения глубины вдавливания $(a)$, пластической работы (б) и твердости (в) при увеличении содержания $\mathrm{Ga}$

Все исследованные образцы с точки зрения фазового состава представляют собой ОЦК неупорядоченный твердый раствор замещения, в котором атомы Ga занимают случайные позиции в решетке $\mathrm{Fe}$. Атомный радиус галлия несколько отличается от атомного радиуса железа, очевидно, что увеличение содержания $\mathrm{Ga}$ усиливает искажения кристаллической решетки и повышает твердость. До 19,1 ат. \% Ga увеличение твердости и уменьшение пластической работы происходит практически линейно в соответствии с законом Вегарда. Однако при переходе от 19,1 \% Ga к 19,7 \% происходит резкий скачок. Твердость возрастает приблизительно на 30 \%, а пластическая работа существенно снижается. Вероятно, это связано с выделением нанокластеров упорядоченной фазы $\mathrm{DO}_{3}$. Так называемая модель структурной неоднородности была предложена в работе [24], авторы предположили, что по мере приближения к границе растворимости $\mathrm{Ga}$ в Fe начинают формироваться нанокластеры $\mathrm{DO}_{3}$ размером не более 15 нм. Согласно данной модели именно с выделением нанокластеров связан первый пик магнитострикции (рис. 1). Для того, чтобы экспериментально обнаружить такие структурные элементы, были проведены специальные эксперименты с использованием метода высокоразрешающей просвечивающей микроскопии (HRTEM) [25] и метода рассеяния нейтронов [26]. Кроме того, сообщается, что нанокластеры не являются идеально кубическими и имеют большую упругую деформацию [26]. В работе наличие нанокластеров напрямую не наблюдалось, но по анализу механических свойств можно сделать вывод о том, 
что существенные макроскопические искажения они начинают вносить при содержании Ga 19,7 \%, что связано с близостью состава к границе растворимости.

\section{4. Заключение}

В работе впервые проанализированы механические свойства сплава Fe-Ga с использованием метода инструментального микроиндентирования. Показано увеличение твердости и уменьшение пластичности с повышением содержания галлия, а также обнаружен резкий скачок свойств при 19,7 ат. \% Ga. Это связывается с искажениями, вносимыми нанокластерами $\mathrm{DO}_{3}$ фазы, о котором сообщалось в более ранних работах. Вероятно, одновременно с положительным влиянием на повышение магнитострикции такие выделения негативным образом сказываются на обрабатываемости сплава.

\section{Благодарность}

Работа выполнена в рамках государственного задания ФАНО России (тема «Магнит» № АAАA-A18-118020290129-5 при частичной поддержке РФФИ (проект № 18-0300623) и комплексной программы УрО РАН № 18-10-2-5.

Экспериментальные результаты получены с использованием оборудования ЦКП «Испытательный цеентр нанотехнологий и перспективных материалов»

\section{Литература}

1. Magnetostrictive properties of body-centered cubic Fe-Ga and Fe-Ga-Al alloys / A. E. Clark, J. B. Restorff, M. Wun-Fogle, T. A. Lograsso, D. L Schlagel // IEEE Transactions on Magnetics. 2000. -Vol. 36. - P. 3238-3240. - DOI: 10.1109/20.908752.

2. Structural investigations of $\mathrm{Fe}-\mathrm{Ga}$ alloys: Phase relations and magnetostrictive behavior / Q. Xing, Y. Du, R. J .McQueeney, T. A. Lograsso // Acta Materialia. - 2008. - Vol. 56. P. 4536-4546. - DOI: 10.1016/j.actamat.2008.05.011.

3. Na S., Flatau A. B. Single grain growth and large magnetostriction in secondarily recrystallized Fe-Ga thin sheet with sharp Goss (011) [100] orientation // Scripta Materialia. - 2012. - Vol. 66. - P. 307-310. - DOI: 10.1016/j.scriptamat.2011.11.020.

4. Magnetostrictive properties of galfenol alloys under compressive stress / A. E. Clark, M. Wun-Fogle, J. B. Restorff, T. A. Lograsso // Materials Transaction. - 2002. - Vol. 43. P. 881-886. - DOI: 10.2320/matertrans.43.881.

5. Temperature and stress dependencies of the magnetic and magnetostrictive properties of Fe 0.81 Ga 0.19 / R. A. Kellogg, A. B. Flatau, A. E. Clark, M. Wun-Fogle, T. A. Lograsso // J. Appl. Phys. - 2002. - Vol. 91. -P. 7821-7823. - DOI: 10.1063/1.1452216.

6. Corrosion studies of single crystals of iron-gallium alloys in aqueous environments / T. V. Jayaraman, N. Srisukhumbowornchai, S. Guruswamy, M. L. Free // Corros. Sci. - 2007. Vol. 49. - P. 4015-4027. - DOI: 10.1016/j.corsci.2007.05.010.

7. Golovin I. S., Riviure A. Mechanisms of anelasticity in Fe-13Ga alloy // Intermetallics. 2011. - Vol. 19. - P. 453-459. - DOI: 10.1016/j.intermet.2010.10.017.

8. Golovin I. S. Anelasticity of Fe-Ga based alloys // Materials \& Design. - 2015. - Vol. 88. P. 577-587. - DOI: 10.1016/j.matdes.2015.08.160.

9. Xing Q., Lograsso T. A. Effect of cooling rate on magnetoelasticity and short-range order in Fe-Ga alloys // Scripta Materialia. - 2011. - Vol. 65. - P. 359-362. DOI: $10.1016 /$ j.scriptamat.2011.05.010.

10. Ductility, texture and large magnetostriction of Fe-Ga-based sheets / J. H. Li, X. X. Gao, J. Zhu, X. Q. Bao, T. Xia, M. C. Zhang // Scripta Materialia. - 2010. - Vol. 63. - P. 246-249. DOI: 10.1016/j.scriptamat.2010.03.068. 
11. Na S., Flatau A. B. Deformation behavior and magnetostriction of polycrystalline Fe-Ga-X $(\mathrm{X}=\mathrm{B}, \mathrm{C}, \mathrm{Mn}, \mathrm{Mo}, \mathrm{Nb}, \mathrm{NbC})$ alloys // Journal of Applied Physics. - 2008. - Vol. 103. P. 07D304. - DOI: 10.1063/1.2838772.

12. Texture evolution and magnetostriction in rolled (Fe81Ga19) $99 \mathrm{Nb} 1$ alloy / J. Li, X. Gao, J. Zhu, C. He, J. Qiao, W. M. Zhang // J. Alloys. Compd. - 2009. - Vol. 476. - P. 529-533. DOI: 10.1016/j.jallcom.2008.09.087.

13. Ductility enhancement and magnetostriction of polycrystalline $\mathrm{Fe}-\mathrm{Ga}$ based alloys / J. Li, X. Gao, J. Zhu, Jie Li, M. Zhang // J. Alloys. Compd. - 2009. - Vol. 484. - P. 203-206. DOI: 10.1016/j.jallcom.2009.03.008.

14. Sun A. L., Liu J. H., Jiang C. B. Microstructural characteristics and in situ reinforcement in NbC-doped Fe81Ga19 magnetostrictive alloys // Materials \& Design. - 2015. - Vol. 88. P. 1342-1346. - DOI: 10.1016/j.matdes.2015.08.150.

15. Takahashi T., Okazaki T., Furuya Y. Improvement in the mechanical strength of magnetostrictive (Fe-Ga-Al)-X-C (X $=\mathrm{Zr}, \mathrm{Nb}$ and $\mathrm{Mo}$ ) alloys by carbide precipitation // Scr. Mater. - 2009. - Vol. 61. - P. 5-7. - DOI: 10.1016/j.scriptamat.2008.12.032.

16. Improved magneostriction and mechanical properties in dual-phase FeGa single crystal / Y. Wu, L. Fang, C. Meng, Y. Chen, J. Wang, J. Liu, T. Zhang, C. Jiang // Materials Research Letters. - 2018. - Vol. 6. - P. 327-332. - DOI: 10.1080/21663831.2018.1451403.

17. Influence of $\mathrm{Tb}$ on structure and properties of $\mathrm{Fe}-19 \% \mathrm{Ga}$ and $\mathrm{Fe}-27 \% \mathrm{Ga}$ alloys / I. S. Golovin, E. M. Balagurov, V. V. Palacheva, A. Emdadi, I. A. Bobrikov, A. Y. Churyumov, V. V. Cheverikin, A. V. Pozdniakov, A. V. Mikhaylovskaya, S. A. Golovin // J. Alloys. Compd. 2016. - Vol. 707. - P. 51-56. - DOI: 10.1016/j.jallcom.2016.09.151.

18. Magnetostriction and microstructure of the melt-spun Fe 83 Ga 17 alloy / M. C. Zhang, H. L. Jiang, X. X. Gao, J. Zhu, S. Z. Zhou // Journal of Applied Physics. - 2006. - Vol. 99. P. 023903. - DOI: 10.1063/1.2164528.

19. Giant magnetostriction on Fe85Ga15 stacked ribbon samples / G. D. Liu, L. B. Liu, Z. H. Liu, M. Zhang, J. L. Chen, J. Q. Li, G. H. Wu // Appl. Phys. Lett. - 2004. - Vol. 84. P. 2124-2126. - DOI: 10.1063/1.1688452.

20. Atulasimha J., Flatau A. B. A review of magnetostrictive iron-gallium alloys // Smart Mater. Struct. - 2011. - Vol. 20. - P. 043001-043001. - DOI: 10.1088/0964-1726/20/4/043001.

21. Magnetic and Mechanical Properties of Polycrystalline Galfenol / E. M. Summers, T. A. Lograsso, J. D. Snodgrass, J. C. Slaughter // Smart Structures and Materials 2004: Active Materials: Behavior and Mechanics : proceedings. - 2004. - Vol. 448. - DOI: 10.1117/12.539781.

22. Phase equilibria and stability of ordered b.c.c. phases in the Fe-rich portion of the $\mathrm{Fe}-\mathrm{Ga}$ system / O. Ikeda, R. Kainuma, I. Ohnuma, K. Fukamichi, K. Ishida // J. Alloys. Compd. - 2002. Vol. 347. - P. 198-205. - DOI: 10.1016/S0925-8388(02)00791-0.

23. Pineau A., Benzerga A. A., Pardoen T. Failure of metals I: Brittle and ductile fracture // Acta Materialia. - 2016. - Vol. 107. - P. 424-483. - DOI: 10.1016/j.actamat.2015.12.034.

24. Khachaturyan A. G., Viehland D. Structurally heterogeneous model of extrinsic magnetostriction for Fe-Ga and similar magnetic alloys: part I. decomposition and confined displacive transformation // Metall. Mater. Trans. A. - 2007. - Vol. 38A - P. 2308-2316. DOI: $10.1007 / \mathrm{s} 11661-007-9253-\mathrm{z}$.

25. Nanodispersed DO3-phase nanostructures observed in magnetostrictive $\mathrm{Fe}-19 \% \mathrm{Ga}$ Galfenol alloys / S. Bhattacharyya, J. R. Jinschek, A. Khachaturyan, H. Cao, J. F. Li, D. Viehland // Phys. Rev. B. - 2008. - Vol. 77. - P. 104107. - DOI: 10.1103/PhysRevB.77.104107.

26. Role of Nanoscale Precipitates on the Enhanced Magnetostriction of Heat-Treated Galfenol $\left(\mathrm{Fe}_{1-\mathrm{x}} \mathrm{Ga}_{\mathrm{x}}\right)$ Alloys / H. Cao, P. M. Gehring, C. P. Devreugd, J. A. Rodriguez-Rivera, J. Li, D. Viehland // Phys. Rev. Lett. - 2009. - Vol. 102. - P. 127201. - DOI: 10.1103/PhysRevLett.102.127201. 